PROCEEDINGS OF THE

AMERICAN MATHEMATICAL SOCIETY

Volume 132, Number 6, Pages 1567-1573

S 0002-9939(04)07433-7

Article electronically published on January 27, 2004

\title{
POSITIVE TERNARY QUADRATIC FORMS WITH FINITELY MANY EXCEPTIONS
}

\author{
WAI KIU CHAN AND BYEONG-KWEON OH \\ (Communicated by David E. Rohrlich)
}

\begin{abstract}
An integral quadratic form $f$ is said to be almost regular if $f$ globally represents all but finitely many integers that are represented by the genus of $f$. In this paper, we study and characterize all almost regular positive definite ternary quadratic forms.
\end{abstract}

\section{INTRODUCTION}

All the quadratic forms considered in this paper are positive definite and have coefficients in $\mathbb{Z}$. A quadratic form $f$ is called almost regular if $f$ represents all but finitely many integers that are represented by gen $(f)$. If $f$ has 5 or more variables, then $f$ is always almost regular; see [12] or [7]. This result would become false for quaternary forms, but it is well known that for every sufficiently large integer $a$ that is represented by the genus of $f$ and is primitively represented by $f_{p}$ for every anisotropic prime of $f, a$ is represented by $f$ itself; see [6] and the references there. Therefore, there are infinitely many inequivalent almost regular primitive quaternary quadratic forms.

There are infinitely many inequivalent almost regular primitive ternary quadratic forms [2, Example 6.2]. A theorem of Watson [14] says that the discriminant of a primitive ternary quadratic form grows with the size of the set of exceptional integers. Hence there are only finitely many inequivalent almost regular primitive ternary quadratic forms if the number of exceptional integers is prescribed. The analytic argument employed by Watson in [14] is not effective, but in this paper we show that for any positive integer $k$, there exists an effective upper bound $C(k)$ for the discriminant of the almost regular ternary quadratic forms with at most $k$ exceptional integers. We also provide a characterization of almost regular ternary quadratic forms (Theorem 4.4), and this should give an effective procedure to recognize almost regular ternary quadratic forms provided that the enumeration of regular ternary quadratic forms is completed. A list containing all possible candidates of regular primitive ternary quadratic forms is available in [8]. This list contains 913 candidates, and all but 22 of them are already verified to be regular.

Received by the editors October 15, 2002.

2000 Mathematics Subject Classification. Primary 11E12, $11 \mathrm{E} 20$.

The research of the first author is partially supported by the National Security Agency and the National Science Foundation.

The work of the second author was supported by KOSEF Grant \# 98-0701-01-05-L. 
The subsequent discussion will be conducted in the more adapted language of quadratic spaces and lattices, and any unexplained notation and terminology can be found in [9] and [10]. The term "ternary lattice" will always refer to an integral $\mathbb{Z}$-lattice on a 3 -dimensional positive definite quadratic space over $\mathbb{Q}$. Since we want to include nonclassic integral quadratic forms in our discussion, it will be assumed throughout this paper that every lattice is even, and in particular $L$ is always an even primitive lattice, i.e., $\mathfrak{n}(L)=2 \mathbb{Z}$. For any lattice $M, Q(M)$ and $Q(\operatorname{gen}(M))$ will denote the sets of integers represented by $M$ and gen $(M)$ respectively. The use of the symbol "*" refers to primitivity. For example, $Q^{*}(M)$ is the set of integers that are primitively represented by $M$. The set $Q(\operatorname{gen}(M)) \backslash Q(M)$ of exceptional integers of $M$ will be denoted by $E(M)$. For the sake of convenience, we set $\delta M=\frac{1}{2} d M$. Since all lattices discussed in this paper are even, $\delta M$ is always an integer. The letter $p$ always denotes a prime in $\mathbb{Z}$.

\section{WATSON'S TRANSFORMATIONS}

Throughout this section, $L$ will denote an even primitive ternary lattice. For any positive integer $m$, let

$$
\Lambda_{m}(L)=\{x \in L: Q(x+z) \equiv Q(z) \bmod m \text { for all } z \in L\}
$$

Let $\lambda_{m}(L)$ be the even primitive lattice obtained from $\Lambda_{m}(L)$ by scaling the ambient space by a suitable rational number. Note that the scaling factor depends on the lattice structure of $L_{p}$ for all $p \mid m$. These $\lambda_{m}$-transformations were used by Watson in his study of regular ternary lattices [13] and class numbers [15], and more recently by various authors in [1] and [2] concerning lattices satisfying different kinds of regularity conditions. These references contain most of the properties of the $\lambda_{m^{-}}$ transformations needed in this paper. We collect a couple of them in the next lemma.

Lemma 2.1. (a) Suppose $L_{p}=M \perp N$ where $M$ is unimodular and $\mathfrak{n}(N) \subseteq 2 p \mathbb{Z}_{p}$. Then $\Lambda_{2 p}(L)_{p}=p M \perp N$.

(b) If $\mathfrak{s}(L)=2 \mathbb{Z}$, then $\Lambda_{4}(L)=\{x \in L: Q(x) \equiv 0 \bmod 4\}$.

Lemma 2.2. If $L$ is almost regular and $\operatorname{ord}_{p}(\delta L) \geq 2$, then $\left|E\left(\lambda_{2 p}(L)\right)\right| \leq|E(L)|$. In particular, $\lambda_{2 p}(L)$ is also almost regular.

Proof. By Lemma 2.1, we can assume that either $p>2$ or $p=2$ but $\mathfrak{s}(L)=\mathbb{Z}$. Then $L_{p}=M \perp N$, where $M$ is (even) unimodular and $\mathfrak{n}(N) \subseteq 4 p \mathbb{Z}_{p}$.

Firstly, let us assume that $M$ is anisotropic. For any $a \in Q\left(\operatorname{gen}\left(\Lambda_{2 p}(L)\right)\right)$, it is clear that $a$ is divisible by $2 p$. If $a \notin E(L)$, then there exists $v \in L$ such that $Q(v)=a$. But then $v \in p M \perp N$, and hence $v \in \Lambda_{2 p}(L)$. In other words, $E\left(\Lambda_{2 p}(L)\right) \subseteq E(L)$.

Suppose $M$ is isotropic. In this case, $L_{p} \cong \mathbb{H} \perp\left\langle 2 p^{\gamma} \epsilon\right\rangle$ for some $\epsilon \in \mathbb{Z}_{p}^{\times}$. Since $\gamma \geq 2$, therefore $\lambda_{2 p}(L)_{p}=\Lambda_{2 p}(L)_{p}^{p^{-2}} \cong \mathbb{H} \perp\left\langle 2 p^{\gamma-2} \epsilon\right\rangle$ and $L_{q} \cong \lambda_{2 p}(L)_{q}$ for all $q \neq p$. As a result, $Q\left(\operatorname{gen}\left(\lambda_{2 p}(L)\right)\right)=Q(\operatorname{gen}(L))$. If $a \in Q\left(\operatorname{gen}\left(\lambda_{2 p}(L)\right)\right)$ and $a \notin E(L)$, then $p^{2} a \in Q\left(\Lambda_{2 p}(L)\right)$ and hence $a \in Q\left(\lambda_{2 p}(L)\right)$.

Remark 2.3. From the proof of the above lemma, it is clear that the conclusion still holds when $p>2 \operatorname{ord}_{p}(d L)=1$ and $\mathbb{Q} L_{p}$ is anisotropic.

Lemma 2.4. Let $L$ be an even primitive almost regular ternary lattice. For any $p$, there exists an even primitive almost regular lattice $\lambda_{(p)}(L)$ such that for any prime 
$q \neq p, \lambda_{(p)}(L)_{q}$ is isometric to $L_{q}$ up to a unit scaling factor and $\operatorname{ord}_{p}\left(\delta\left(\lambda_{(p)}(L)\right)\right) \leq$ 1.

Proof. Note that $\operatorname{ord}_{p}\left(\delta\left(\lambda_{2 p}(L)\right)\right)<\operatorname{ord}_{p}(\delta L)$ if the latter is at least 2 [1, Lemma 2.5 . Hence the lattice $\lambda_{(p)}(L)$ can be obtained from $L$ by means of a finite number of $\lambda_{2 p}$-transformations.

Lemma 2.5. Suppose that $\mathbb{Q} L_{p}$ is anisotropic. There exists an integer $k=k(L)$ such that for any $a \in Q(\operatorname{gen}(L))$ that is divisible by $p^{k}, a \in Q(L)$ if and only if $p^{-k} a \in Q\left(\lambda_{(p)}(L)\right)$.

Proof. First of all, since $\mathbb{Q} L_{p}$ is anisotropic, it is not hard to see that $\Lambda_{2 p}(L)$ is simply the set $\{x \in L: Q(x) \equiv 0 \bmod 2 p\}$. Set $L(0)=L$ and for any $n \geq 1$, let $L(n)$ be the lattice $\lambda_{2 p}(L(n-1))$. Let $t$ be the smallest integer such that $L(t)=\lambda_{(p)}(L)$. If $t=0$, then we can choose $k=0$. Otherwise, for any $t-1 \geq n \geq 0$, let $k_{n}$ be the integer such that $\Lambda_{2 p}(L(n))^{p^{-k_{n}}}=\lambda_{2 p}(L(n))$. Note that $k_{n}$ is either 1 or 2 . Put

$$
k=k_{0}+\cdots+k_{t-1} .
$$

If $p^{k} \mid a$ and $p^{-k} a \in Q\left(L_{t}\right)$, then $p^{k_{t-1}-k} a$ is represented by $\Lambda_{2 p}(L(t-1)) \subseteq L(t-1)$. Apply the same argument repeatedly until we have $a \in Q(L)$.

Conversely, if $a \in Q(L)$ and $p^{k} \mid a$, then $a \in Q\left(\Lambda_{2 p}(L(0))\right)$ and hence $p^{-k_{0}} a \in$ $Q(L(1))$. Repeating this argument, we see that $p^{-k} a \in Q(L(t))$.

\section{Almost Regular ternary lattices}

Let $S_{L}$ be the set of primes $p$ for which $\mathbb{Q} L_{p}$ is anisotropic.

Lemma 3.1. If $L$ is almost regular and $p \in S_{L}$, then $\lambda_{(p)}(L)$ is regular. In particular, if $L=\lambda_{(p)}(L)$, then $L$ is regular.

Proof. Let us assume to the contrary that $\lambda_{(p)}(L)$ is not regular for some $p \in S_{L}$. If $a \in E\left(\lambda_{(p)}(L)\right)$, then $p^{2 m} a \in E\left(\lambda_{(p)}(L)\right)$ for all $m \geq 0$. This contradicts the almost regularity condition of $\lambda_{(p)}(L)$.

Lemma 3.2. Suppose $\lambda_{(p)}(L)$ is regular for every $p \in S_{L}$. If $Q^{*}(\operatorname{gen}(L)) \backslash Q(L)$ is a finite set, then $L$ is almost regular.

Proof. Let $a$ be an integer in the set $Q(\operatorname{gen}(L)) \backslash\left(Q^{*}(\operatorname{gen}(L)) \cup Q(L)\right)$. By Lemma 2.5 we see that $\operatorname{ord}_{p}(a)$ is bounded for all $p \in S_{L}$. If $q \notin S_{L}$, then $Q^{*}\left(L_{q}\right)$ contains an ideal $q^{\gamma_{q}} \mathbb{Z}_{q}$ where $\gamma_{q}$ depends only on $L$ and $\gamma_{q}=0$ if $q \nmid 2 d L$. Therefore, we can write $a=\alpha^{2} \beta$ where $\alpha$ is only divisible by the prime divisors of $2 d L$ and $\beta \in Q^{*}(\operatorname{gen}(L))$. Furthermore, $\alpha$ is bounded by a constant depending only on $L$. Since $Q^{*}(\operatorname{gen}(L)) \backslash Q(L)$ is finite, only finitely many of those $\beta$ are not in $Q(L)$ and hence $L$ is almost regular.

Let $P_{L}$ be the set of all primitive elements [11, p. 353] of $Q^{*}(\operatorname{gen}(L))$ that are primitive spinor exceptions of $\operatorname{gen}(L)$. This set is finite and can be determined by the results in [5].

Theorem 3.3. A ternary lattice $L$ is almost regular if and only if $L$ represents all integers in $P_{L}$ and $\lambda_{(p)}(L)$ is regular for all $p \in S_{L}$. 
Proof. Suppose $L$ is almost regular. We already showed in Lemma 3.1 that $\lambda_{(p)}(L)$ is regular for all $p \in S_{L}$. Assume that there exists $a \in P_{L}$ that is not represented by $L$. Then there exist infinitely many primes $q$ such that $a q^{2}$ is not primitively represented by $\operatorname{spn}^{+}(L)$ [4 Theorem 1]. However, $L$ must represent one of these integers, say $a q_{0}^{2}$ where $q_{0} \nmid 2 d L$, and hence there exists an integer $d>1$ such that $\frac{a q_{0}^{2}}{d^{2}}$ is in $Q^{*}(L)$. Since $a \notin Q(L), q_{0}$ cannot divide $d$ and thus $\frac{a}{d^{2}}$ is an integer. Since $L_{q_{0}}$ is isotropic, $\frac{a}{d^{2}}$ is in $Q^{*}(\operatorname{gen}(L))$, which contradicts the fact that $a \in P_{L}$.

For the converse, we may assume that $a \in Q^{*}(\operatorname{gen}(L))$ by virtue of Lemma 3.2 If $a$ is primitively represented by $\operatorname{spn}^{+}(L)$, then $a \in Q(L)$ if $a$ is sufficiently large 3. So, we may further assume that $a$ is a primitive spinor exceptional integer. Let $t$ be a primitive element of $Q^{*}(\operatorname{gen}(L))$ such that $a=t m^{2}$ for some $m \geq 1$. This $t$ is bounded because there are only finitely many primitive elements of $Q^{*}(\operatorname{gen}(L))$ that are in one of those primitive spinor exceptional square classes [11. If $t \in P_{L}$, then $a \in Q(L)$ and we are done. Therefore, we may assume that $t \notin P_{L}$ and hence $t$ is not a primitive spinor exception of gen $(L)$. Then for any sufficiently large prime $q, t q^{2}$ is also not a primitive spinor exception [5] and hence $t q^{2}$ is in $Q(L)$ [6, Corollary 4.3]. As a result, we may assume that the prime divisors of $m$ are bounded. It is well known that if $h$ is the number of classes in $\operatorname{spn}^{+}(L)$ and $\mathbb{Q} L_{q}$ is isotropic, then $t m^{2}$ is already represented by $L$ provided that $q^{h} \mid m$. If $p \in S_{L}, \operatorname{ord}_{p}(a)$ is bounded because $a$ is in $Q^{*}(\operatorname{gen}(L))$. Therefore, the integers in $Q^{*}(\operatorname{gen}(L)) \backslash Q(L)$ are bounded, and hence $L$ is almost regular.

\section{Characterization of almost Regularity}

Let $L$ be an almost regular ternary lattice. It is easy to see that $\mathbb{Q} L_{p}$ is isotropic if and only if the Hasse invariant of $\mathbb{Q} L_{p}$ is equal to $(-1,-1)_{p}$. By the Hilbert Reciprocity Law it follows that the set $S_{L}$ must contain an odd number of primes. In particular, $S_{L}$ is never empty. Therefore, by Lemma 3.1, the prime divisors of $d L$ must occur as divisors of the discriminants of some regular ternary lattices, and they are inside $\{2,3,5,7,11,13,17,23\}$; see [8] or 13]. If $p \in S_{L}$, then for any $q \neq p, \operatorname{ord}_{q}(d L)$ must be bounded above by the bounds given by Watson [13. If $S_{L}$ contains a prime other than $p$, then $\operatorname{ord}_{p}(d L)$ would also be bounded by Watson's bounds. In other words, the number of classes of even primitive almost regular ternary lattices with at least 3 anisotropic primes is finite. In this section we are going to show that these lattices are, in fact, regular.

We will rely heavily on the table of regular ternary lattices in $[8$, and the readers are advised to have that in hand. In that table, an array $[a, b, c, d, e, f]$ represents a primitive quadratic form $g(x, y, z)=a x^{2}+b y^{2}+c z^{2}+d y z+e x z+f x y$. The form $2 g$ corresponds to an even primitive lattice $L$. Under this correspondence, $g$ is an odd form if and only if $\mathfrak{s}(L)=\mathbb{Z}$. In what follows, we will simply say that $L$ is $[a, b, c, d, e, f]$ if $L$ corresponds to $2 g(x, y, z)$.

Lemma 4.1. Let $L$ be an almost regular ternary lattice and $p \in S_{L}$. If $Q^{*}\left(L_{p}\right) \subseteq$ $2 \mathbb{Z}_{p}^{\times} \cup 2 p \mathbb{Z}_{p}^{\times}$, then $L$ is regular.

Proof. It is clear: if $a \in E(L)$, then $p^{2 n} a \in E(L)$ for all $n \geq 0$.

Remark 4.2. Lemma 4.1 applies when $p$ is odd and $L_{p} \cong\langle\epsilon\rangle \perp\langle p,-\Delta p\rangle$ where $\epsilon \in \mathbb{Z}_{p}^{\times}$, or when $p=2$ and $L_{2}$ is modular. 
For any even ternary lattice $L$, let $\lambda(L)$ be the even primitive ternary lattice obtained by applying various $\lambda_{2 p}$-transformations successively to $L$ until $\operatorname{ord}_{p}(\delta(\lambda(L)))$ is at most 1 for all $p$.

Theorem 4.3. Let $L$ be an almost regular ternary lattice. If $\left|S_{L}\right| \geq 3$, then $L$ is regular.

Proof. For any $p \in S_{L}, \lambda(L)_{p}$ is anisotropic and hence $\operatorname{ord}_{p}(\delta(\lambda(L)))=1$. From [8] or [16, 17, we see that $d(\lambda(L))=4 p q$, where $(p, q)=(3,5),(3,7),(5,7)$, or $(3,13)$. Suppose $L$ is not regular. Then by Remark 4.2 we may assume that both $\operatorname{ord}_{p}(d L)$ and $\operatorname{ord}_{q}(d L)$ are at least 3. The lattice $\lambda_{(2)}(L)$ is regular, and its discriminant is divisible by $4 p^{3} q^{3}$. From $[8$, the only possible candidate is $[3,17,35,5,0,3]$ with discriminant $4 \cdot 3^{3} \cdot 5^{3}$; the other candidates are ruled out since they are isotropic at either $p$ or $q$. By Remark $2.3 \lambda_{\ell}\left(\lambda_{(\ell)}(L)\right)$ is regular for $\ell=3,5$.

If $\mathfrak{s}(L)=\mathbb{Z}$, then the discriminant of $\lambda_{3}\left(\lambda_{(3)}(L)\right)$ must be divisible by $16 \cdot 3^{2} \cdot 5^{3}$. But there is no regular ternary lattice with this discriminant [8]. If $\mathfrak{s}(L)=2 \mathbb{Z}$, then $L$ is not modular and $d L$ must be of the form $2^{s} \cdot 3^{3} \cdot 5^{3}$ for some $s \geq 4$. Using [8], we find out that $\lambda_{3}\left(\lambda_{(3)}(L)\right)$ could only be

$$
[15,24,56,24,0,0] \text { or }[8,15,152,0,8,0]
$$

and hence $d\left(\lambda_{3}\left(\lambda_{(3)}(L)\right)\right)=2^{7} \cdot 3^{2} \cdot 5^{3}$. This also shows that $d L=2^{7} \cdot 3^{3} \cdot 5^{3}$.

Now, let us consider $\lambda_{5}\left(\lambda_{(5)}(L)\right)$. Its discriminant should be $2^{7} \cdot 3^{3} \cdot 5^{2}$. There are only three possible candidates:

$$
[11,26,39,6,6,2], \quad[11,35,39,-30,6,10], \quad[9,41,41,-38,6,6] .
$$

By comparing the 2-adic structures of $\lambda_{3}\left(\lambda_{(3)}(L)\right)$ and $\lambda_{5}\left(\lambda_{(5)}(L)\right)$, we can conclude that $\lambda_{5}\left(\lambda_{(5)}(L)\right)$ must be $[9,41,41,-38,6,6]$. Since $L_{5}$ is of the form $\left\langle a, 5 b, 5^{2} c\right\rangle$ for some $a, b, c \in \mathbb{Z}_{5}^{\times}$, therefore $L_{3}^{5}=\lambda_{5}\left(\lambda_{(5)}(L)\right)_{3}$ and thus

$$
L_{3} \cong\langle\Delta\rangle \perp\langle 3\rangle \perp\left\langle 3^{2} \Delta\right\rangle \text {. }
$$

By a similar token, we have

$$
L_{2} \cong\langle 10\rangle \perp \mathbb{A}^{8} \quad \text { and } \quad L_{5} \cong\langle\Delta \epsilon\rangle \perp\langle 5 \Delta\rangle \perp\left\langle 5^{2} \epsilon\right\rangle,
$$

where $\epsilon \in \mathbb{Z}_{5}^{\times}$. All these together show that $L$ is in the genus of $[13,28,157,28,2,4]$ or $[24,45,76,40,12,0]$. But both genera have class number 1 [8] and hence $L$ is regular, which is a contradiction.

The following theorem summarizes all the results we have obtained thus far.

Theorem 4.4. Let $L$ be a ternary lattice that is not regular. Then $L$ is almost regular if and only if all of the following conditions hold:

(1) $S_{L}=\{p\}$ where $p \in \mathcal{S}=\{2,3,5,7,11,13,17\}$;

(2) $\lambda_{(p)}(L)$ is regular;

(3) $L$ represents all integers in $P_{L}$.

Remark 4.5. The set $\mathcal{S}$ defined above does not contain 23 because every regular ternary lattice is isotropic at 23; see [8] or [16, 17] (also the appendix in [1]).

Example 4.6. For any $n \geq 0$, let $K(n)$ be the lattice $\langle 1\rangle \perp\langle 1\rangle \perp\left\langle 3^{2 n+1}\right\rangle$. In [2], it was shown that $K(n)$ is almost regular for each $n$. Theorem 4.4 gives us an easy way to verify this fact. For, gen $(K(n))$ has only one spinor genus and hence $P_{L}$ is empty. Furthermore, $S_{L}=\{3\}$ and $K(0)$ is regular from 8 . 


\section{DisCRIMINANT BOUNDS}

Theorem 5.1. Given any integer $k \geq 0$, there exists a computational effective upper bound for the discriminants of even primitive ternary lattices $L$ for which the size of the exceptional set $E(L)$ does not exceed $k$.

Proof. Let $L$ be such a lattice. We may assume that $L$ is not regular and hence $S_{L}=\{p\} \subset \mathcal{S}$. Since $\lambda_{(p)}(L)$ is regular, $\operatorname{ord}_{q}(d L)$ is bounded for any $q \neq p$, and an explicit bound can be found in [13] or deduced from [8]. Therefore, it suffices to show that $\operatorname{ord}_{p}(d L)$ is bounded by a constant depending only on $k$ and $p$. By Lemma 2.2. we may further assume that $\operatorname{ord}_{q}(\delta L) \leq 1$ for all $q \neq p$. In particular, $Q\left(L_{q}\right)=2 \mathbb{Z}_{q}$ for all $q \neq p$.

Since $L$ is almost regular and $\mathfrak{n}(L)=2 \mathbb{Z}$, the set $Q(L) \cap 2 \mathbb{Z}_{p}^{\times}$contains infinitely many integers. So we can find two integers in this set, independent of $L$, to serve as the bounds for the first and the second minima of $L$. Let $\mathcal{B}$ be the collection of positive binary lattices whose successive minima are less than those bounds. For each lattice $N \in \mathcal{B}$, select an integer from $Q(L) \backslash Q(N)$. Such an integer can be chosen to be independent of $L$. Since $\mathcal{B}$ is finite, therefore the third minimum of $L$ is also bounded by a constant depending only on $k$.

The above sets forth an effective procedure to bound $\operatorname{ord}_{p}(d L)$, although in practice it could involve laborious computations. Below, we illustrate the effectiveness by exhibiting a priori bounds for $\operatorname{ord}_{p}(d L)$ when $|E(L)|=1$.

Theorem 5.2. Let $L$ be an almost regular ternary lattice with $|E(L)|=1$. Suppose $S_{L}=\{p\}$ where $p$ is an odd prime and $L_{p} \cong\left\langle a, p^{\beta} b, p^{\gamma} c\right\rangle$, where $0 \leq \beta \leq \gamma$ are integers and $a, b, c \in \mathbb{Z}_{p}^{\times}$.

(a) If $\beta=0$, then $p \leq 5$ and

$$
\gamma= \begin{cases}3 & \text { if } p=5, \\ 3 \text { or } 5 & \text { if } p=3 .\end{cases}
$$

(b) If $\beta=1$, then $p \leq 7$ and

$$
2 \leq \gamma \leq \begin{cases}2 & \text { if } p=7 \\ 3 & \text { if } p=5, \\ 5 & \text { if } p=3 .\end{cases}
$$

(c) If $\beta>1$, then $p=3, \beta \leq 3$ and $\beta+\gamma \leq 6$.

Theorem 5.3. Let $L$ be an almost regular ternary lattice with $|E(L)|=1$. Suppose $S_{L}=\{2\}$ and $L_{2} \cong M \perp\left\langle 2^{\gamma} c\right\rangle$.

(a) If $M \cong A(2,2)$, then $\gamma \leq 8$.

(b) Suppose $M \cong\left\langle 2 a, 2^{\beta} b\right\rangle$ for some $a, b \in \mathbb{Z}_{2}^{\times}$and $1 \leq \beta \leq \gamma$. Then

(i) if $\beta=1$, then $\gamma \leq 11$;

(ii) if $\beta=2$, then $\gamma \leq 9$;

(iii) in general, $\beta \leq 9$ and $\gamma \leq 20$.

The proofs of the above theorems will not be given since the general idea and method are already clear. But we do want to make a few comments here. The set $Q(L) \cap 2 \mathbb{Z}_{p}^{\times}$often contains small integers, and hence the collection $\mathcal{B}$ is usually not too big. Moreover, a lot of candidates in $\mathcal{B}$ can be ruled out fairly quickly by 
considering their local structures. In Theorem5.3(b)(iii), we just need to determine the bounds for the first two minima. For, $2^{\beta+1}$ is always less than the product of the first two minima. Furthermore, we can apply the $\lambda_{4}$-transformation a finite number of times to $L$ and obtain a lattice $K$ so that

$$
K_{2} \cong \begin{cases}\left\langle 2 a^{\prime}, 2 b^{\prime}, 2^{\gamma-\beta+1} c^{\prime}\right\rangle & \text { if } \beta \text { is odd; } \\ \left\langle 2 a^{\prime}, 4 b^{\prime}, 2^{\gamma-\beta+2} c^{\prime}\right\rangle & \text { otherwise }\end{cases}
$$

where $a^{\prime}, b^{\prime}, c^{\prime} \in \mathbb{Z}_{2}^{\times}$. Therefore, $\gamma-\beta$ is bounded by parts (b)(i) and (b)(ii).

\section{REFERENCES}

1. W. K. Chan and A. G. Earnest, Discriminant bounds for spinor regular ternary quadratic lattices, submitted.

2. W. K. Chan and B.-K. Oh, Finiteness theorems for positive definite n-regular quadratic forms, Trans. Amer. Math. Soc., 355 (2003), 2385-2396.

3. W. Duke and R. Schulze-Pillot, Representations of integers by positive ternary quadratic forms and equidistribution of lattice points on ellipsoids, Invent. Math., 99 (1990), 49-57. MR 90m:11051

4. A. G. Earnest, Representations of spinor exceptional integers by ternary quadratic forms, Nagoya Math. J., 93 (1984), 27-38. MR 85j:11042

5. A. G. Earnest, J. S. Hsia and D. C. Hung, Primitive representations by spinor genera of ternary quadratic forms, J. London Math. Soc. (2), 50 (1994), 222-230. MR 95k:11044

6. J. S. Hsia and M. Jöchner, Almost strong approximations for definite quadratic spaces, Invent. Math., 129 (1997), 471-487. MR 98m:11025

7. J. S. Hsia, Y. Kitaoka and M. Kneser, Representations of positive definite quadratic forms, J. Reine Angew. Math., 301 (1978), 132-141. MR 58:27758

8. W. C. Jagy, I. Kaplansky and A. Schiemann, There are 913 regular ternary forms, Mathematika, 44 (1997), 332-341. MR 99a:11046

9. Y. Kitaoka, Arithmetic of quadratic forms, Cambridge University Press, 1993. MR 95c:11044

10. O. T. O'Meara, Introduction to quadratic forms, Springer-Verlag, New York, 1963. MR 27:2485

11. R. Schulze-Pillot, Exceptional integers for genera of integral ternary positive definite quadratic forms, Duke Math. J., 102, No. 2 (2000), 351-357. MR 2001a:11068

12. W. Tartakowsky, Die Gesamtheit der Zahlen, die durch eine positive quadratische Form $F\left(x_{1}, \ldots, x_{s}\right)(s \geq 4)$ darstellbar sind, Izv. Akad. Nauk SSSR, 7 (1929), 111-122, 165-195.

13. G. L. Watson, Some problems in the theory of numbers, Ph.D. Thesis, University of London, 1953.

14. G. L. Watson, The representation of integers by positive ternary quadratic forms, Mathematika 1 (1954), 104-110. MR 16:680c

15. G. L. Watson, The class-number of a positive quadratic form, Proc. London Math. Soc. 13 (1963), 549-576. MR 27:107

16. G. L. Watson, One-class genera of positive ternary quadratic forms, Mathematika, 19 (1972), 96-104. MR 47:3317

17. G. L. Watson, Regular positive ternary quadratic forms, J. London Math. Soc. (2) 13 (1976), 97-102. MR 54:2590

Department of Mathematics, Wesleyan University, Middletown, Connecticut 06459

E-mail address: wkchan@wesleyan.edu

Department of Applied Mathematics, Sejong University, Seoul 143-747, Korea

E-mail address: bkoh@sejong.ac.kr 